\title{
Isolation of Propionibacterium acnes among the microbiota of primary endodontic infections with and without intraoral communication
}

\author{
Sadia Ambreen Niazi ${ }^{1,2}$ • Hana Suleiman Al Kharusi ${ }^{1}$ - Shanon Patel ${ }^{1}$. \\ Kenneth Bruce $^{2,3}$ • David Beighton ${ }^{2} \cdot$ Federico Foschi $^{1,4} \cdot$ Francesco Mannocei $^{1,4}$
}

Received: 25 November 2015 / Accepted: 24 January 2016/Published online: 9 February 2016

(C) The Author(s) 2016. This article is published with open access at Springerlink.com

\begin{abstract}
Objectives The presence of opportunistic pathogens such as Propionibacterium acnes ( $P$. acnes) may contribute to the endodontic pathology. The presence of $P$. acnes may be influenced by different endodontic conditions.

The aims of the study were firstly, to identify $P$. acnes within the whole cultivable microbiota of primary endodontic infections, to investigate which $P$. acnes phylotypes predominate in such infections and secondly to determine if the presence of an "open" communication (e.g. a sinus) can be associated with the isolation of $P$. acnes from the root canal.

Material and methods The predominant cultivable microbiota of 15 primary endodontic lesions (7 without communication with the oral environment and 8 with an open communication) were identified using partial 16S ribosomal RNA (rRNA) gene sequence analysis. The identification of the organism was determined by interrogating the Human Oral Microbiome Database. The P. acnes isolates were typed on
\end{abstract}

Federico Foschi

federico.foschi@kcl.ac.uk

1 Department of Restorative Dentistry, King's College London Dental Institute at Guy's, King's and St Thomas' Hospital, Floor 22, Tower Wing, Guy's Hospital, St Thomas' St, London SE1 9RT, UK

2 Department of Microbiology, King's College London Dental Institute at Guy's, King's and St Thomas' Hospital, Floor 17, Tower Wing, Guy's Hospital, St Thomas' St, London SE1 9RT, UK

3 Institute of Pharmaceutical Science, King's College London, Floor 5, 150 Stamford Street, London SE1 9NH, UK

4 Biomaterials, Biomimetics and Biophotonics Group, King's College London Dental Institute at Guy's, King's and St Thomas' Hospital, Floor 17, Tower Wing, Guy's Hospital, St Thomas' St, London SE1 9RT, UK the basis of the recA gene sequence comparison. A neighborjoining tree was constructed using MEGA 4.1 with the inclusion of known recA sequences.

Results There was no difference in the number of species identified from lesions without communication $(5.86 \pm 3.7)$ and those with communication $(5.37 \pm 3.6)(P>0.05)$. PCRbased 16S rRNA gene sequencing revealed $P$. acnes as the most prevalent isolate recovered from lesions with communication. recA gene sequencing revealed two phylogenetic lineages present in lesion with communication, with mainly type I (further split into type IA and type IB) and type II.

Conclusions The presence of $P$. acnes as opportunistic pathogens has been confirmed and may sustain the traits observed in specific clinical presentations.

Clinical relevance Clinical management of open lesions may require further disinfection to eliminate opportunistic bacteria.

Keywords P.acnes $\cdot$ Endodontic disease $\cdot$ Nosocomial infection · Fistula

\section{Introduction}

Propionibacterium acnes (P. acnes) is a Gram-positive anaerobic/aerotolerant rod that is a resident microbiota of skin, oral cavity, large intestine, conjunctiva and external ear canal [1-4]. Recent studies have identified P. acnes as an opportunistic pathogen linked to a wide range of infections and inflammatory conditions including acnes vulgaris $[5,6]$, sarcoidosis [7], synovitis-acnes-pustulosis-hyperostosis-osteitis syndrome $[8,9]$ and prostate cancer [10]. The P. acnes infections are also linked with trauma and surgery including brain abscesses [11], osteomyelitis after lumbar puncture [12], discitis after surgery [13], spodylodiscitis following epidural catheterization [14], post-operative mediastinitis [15], 
endophthalmitis [16] and endocarditis [17]. Moreover, foreign body implants are one of the predisposing factors associated with $P$. acnes infections [10, 12, 14].

$P$. acnes have been classified into four highly distinct evolutionary lineages: type IA, IB, II and III, which display differences in inflammatory properties, production of virulence determinants and association with various conditions [18-23]. Type I is usually associated with skin colonization [18], whereas type II and III are almost exclusively associated with infections of implanted prosthesis [19, 24] suggesting their possible role in the pathogenesis of the implant infections. A previous study concluded that $P$. acnes as an opportunistic pathogen was associated much more often than previously reported, with apical periodontitis in teeth with an existing root canal treatment (secondary endodontic infection) [25].

Bacteria may penetrate into the root canal space via caries, trauma and also during the root canal treatment procedure itself due to poor cross infection control and/or inadequate isolation of the operatory field [26]. It has been shown that $P$. acnes type II and III are associated with refractory endodontic infections [25]. It is possible that $P$. acnes may be introduced into the root canal at the time of the root canal obturation or may infect the root canal post-treatment as a result of bacterial leakage at the tooth-restoration interface [25, 27].

The ecology of primary endodontic infection is unique, harboring microbiota, which depend on the presence or absence of a frank communication with the oral cavity. Variation in oxygen tension, availability of metabolites/substrates and replenishment of the biofilm via continuous re-colonization through "open" communications with the oral cavity associated with the sinus tract, full depth periodontal pocket and a history of trauma (resulting in root fracture, tooth avulsion or subluxation) may all significantly alter the microbiota present in primary endodontic infections [26].

The primary aim of the present study was to determine if $P$. acnes is present within the whole cultivable microbiota of primary endodontic infections. The secondary aims were to investigate which specific recA phylotypes of $P$. acnes predominate in such infections, and finally to determine if the presence of an "open" communication with the endodontic niche can be associated with a more frequent isolation of $P$. acnes from the root canal space.

\section{Methods}

\section{Patients selections}

Patients recruited into the study received treatment in the Endodontic Department of the Dental Institute at Guys' and St Thomas' Hospital, King's College London. The project was approved by the local ethics committee (South London REC: 05/Q0705/051). The patient had verbal and written information about the purpose of the study, and they gave their written informed consent prior to their inclusion. The selected teeth were single rooted with necrotic pulp (primary lesions). Samples were assigned according to clinical history to group A (absence [Table 1]), or group B (presence [Table 2]) of a communication with the oral environment. In group A, $(n=7)$ samples were collected from the root canal system from teeth which had no macroscopic communication between the tooth and the oral cavity (Table 1$)$. In group B, $(n=8)$ samples were collected from the root canal system from teeth that had evidence of communication with the oral environment either through the presence of a sinus tract, periodontal probing depths of at least $8 \mathrm{~mm}$ and/or recent history of trauma resulting in subluxation, luxation and horizontal root fracture in the presence of deep periodontal pocketing (Table 2). All patients were healthy with no active, acute or chronic medical conditions and had not been prescribed antibiotics during the previous month. All significant details about the dental history, clinical and radiographic signs of the involved tooth were recorded (Tables 1 and 2).

\section{Samples collection}

After local anesthesia, the tooth to be treated was isolated with the rubber dam. The tooth the clamp and the surrounding dam were cleaned with $30 \%(v / v)$ hydrogen peroxide and decontaminated with $2.5 \%$ sodium hypochlorite followed by sodium thiosulphate. After decontamination, the isolated tooth and surrounding dam were swabbed to check for contamination. The existing restoration and caries were removed with a sterile bur, after which the access cavity was completed with a new sterile high-speed carbide bur without watercooling until the pulp chamber was exposed. The content of the root canal space was removed using a combination of sterile K-type or Hedstrom files (Dentsply Maillefer, Baillagues, Switzerland) as well as rotary instruments (ProTaper, Dentsply Maillefer) without any irrigation. All the tissues removed were transferred into $1 \mathrm{ml}$ PRAS medium (Oxyrase, Mansfield, OH, USA). All samples were immediately transported on ice to the laboratory, after which endodontic treatment was completed.

\section{Microbial analysis of samples}

Each sample was dispersed by vortexing with sterile glass beads, diluted in fastidious anaerobe broth (Lab M, UK) and plated onto non-selective media; duplicate plates of fastidious anaerobe agar (FAA) supplemented with $5 \%$ horse blood (Lab M, UK). The FAA plates were incubated anaerobically for 7 days. After incubation, colonies were counted, and predetermined number of colonies ( $n=30$ per patient) were randomly selected by using the grid to divide the plate into 
Table 1 Group A samples without communication

\begin{tabular}{llllllll}
\hline Sample & Sex & $\begin{array}{l}\text { Age } \\
\text { (Year) }\end{array}$ & $\begin{array}{l}\text { Coronal } \\
\text { restoration }\end{array}$ & $\begin{array}{l}\text { Periodontal } \\
\text { pockets }(\mathrm{mm})\end{array}$ & Clinical findings & Tooth & Tx provided \\
\hline 1 & M & 30 & - & 2 & Necrotic discolored PA lesion & UL1 & RCT bleaching \\
2 & F & 24 & Amalgam & 1 & Deep restoration, necrotic PA lesion & LR5 & RCT PFM crown \\
3 & F & 19 & Composite & 1 & Old trauma Necrotic PA lesion & UR2 & RCT bleaching \\
4 & M & 42 & - & 3 & Enamel infraction necrotic discolored & UR1 & RCT bleaching \\
5 & F & 27 & IRM & 2 & Deep cavity failed pulp capping & LL3 & RCT ceramic crown \\
6 & F & 35 & Composite & 2 & Necrotic discolored PA lesion & LR2 & RCT Composite core \\
7 & M & 20 & Composite & 1 & Deep MOD filling necrotic & UL4 & RCT fiber post-composite core \\
8 & M & 11 & - & 2 & Old trauma necrotic discolored PA lesion symptomatic & UR1 & RCT bleaching \\
\hline
\end{tabular}

different sections for gram-staining and molecular identification.

The swabs taken from the prepared teeth prior to the removal of the restoration were plated directly onto FAA and incubated anaerobically for 7 days.

\section{Identification of isolates}

All randomly selected isolates were subcultured on FAA plates and grown anaerobically for $24 \mathrm{~h}$. Bacterial genomic DNA was extracted by boiling $100 \mu \mathrm{l}$ of a suspension of the cultured cells prepared in sterile distilled $\mathrm{H}_{2} \mathrm{O}$ for $10 \mathrm{~min}$, followed by cooling on ice for $10 \mathrm{~min}$ and centrifuged at $13,000 \times \mathrm{g}$ for $2 \mathrm{~min}$ [18]. The supernatant containing the genomic DNA was stored at $-20{ }^{\circ} \mathrm{C}$ prior to analysis. DNA amplification of a partial fragment of the 16S ribosomal (rRNA) gene of the isolates was performed using universal primers: 9F (5'-3' GAGTTTGATCCTGGCTCA) and 907R (5'-3' CGTCAATTCCTTTGAGTT) [28]. The amplification was carried out with the following reaction mixture (final volume, $25 \mu \mathrm{l}): 0.5 \mu \mathrm{l}$ of $9 \mathrm{~F}$ forward primers (concentration $10 \mathrm{pmol} /$ $\mu \mathrm{l}$; MWG, UK), $0.5 \mu \mathrm{l}$ of 907R reverse primer (concentration $10 \mathrm{pmol} / \mu \mathrm{l}$; MWG), $23 \mu \mathrm{l}$ of Reddymix buffer (Thermo
Scientific, UK) and $1 \mu \mathrm{DNA}$ extract. The thermal cycling conditions included initial denaturation at $94{ }^{\circ} \mathrm{C}$ for $10 \mathrm{~min}$, denaturation at $94{ }^{\circ} \mathrm{C}$ for $30 \mathrm{~s}$, annealing at $49{ }^{\circ} \mathrm{C}$ for $30 \mathrm{~s}$ and extension at $72{ }^{\circ} \mathrm{C}$ for $90 \mathrm{~s}$, repeated for 34 cycles and final extension at $72{ }^{\circ} \mathrm{C}$ for $5 \mathrm{~min}$. The amplified products were run on a $0.5 \%$ agarose gel (Sigma, UK) and visualized under UV transillumination. PCR products were cleaned for the sequence reaction with Microclean (Sigma, UK) according to the manufacturer's instructions.

Amplicon sequencing was performed by using an ABI Prism BigDye Terminator sequencing kit (Applied Biosystems) with 30 cycles of denaturation at $96^{\circ} \mathrm{C}$ for $10 \mathrm{~s}$, annealing at $50{ }^{\circ} \mathrm{C}$ for $5 \mathrm{~s}$ and extension at $60^{\circ} \mathrm{C}$ for 2 min. Sequencing reaction products were run on an $\mathrm{ABI}$ 3730xl sequencer Applied Biosystems). All DNA sequences were analyzed, trimmed and aligned using BioEdit software (version 7.0.0; http://www.mbio.ncsu.edu/BioEdit/bioedit. html). The partial gene sequences were identified by a BLAST search of the NCBI database (http://0-www.ncbi. nlm.nih.gov.ilsprod.ilb.neu.edu/BLAST/), the Human Oral Microbiome database (http://www.homd.org/) or the Ribosome Database Project database http://rdp.cme.msu. edu/). Phylogenetic trees were constructed by the neighbor-

Table 2 Group B samples with communication

\begin{tabular}{llllllll}
\hline Sample & Sex & $\begin{array}{l}\text { Age } \\
\text { (Year) }\end{array}$ & $\begin{array}{l}\text { Coronal } \\
\text { restoration }\end{array}$ & $\begin{array}{l}\text { Periodontal } \\
\text { pockets }(\mathrm{mm})\end{array}$ & Clinical findings & Tooth & Tx provided \\
\hline 9 & F & 21 & - & $5+$ & $\begin{array}{l}\text { Horizontal root fracture, deep periodontal } \\
\text { pocket communicating with the fracture }\end{array}$ & UR1 & RCT MTA Composite build-up \\
10 & F & 23 & Composite & 3 & Necrotic abscess sinus symptomatic & UR2 & RCT bleaching composite build-up \\
11 & M & 15 & - & 3 & Trauma avulsed surgically repositioned and splinted & UR1 & RCT bleaching composite build-up \\
12 & M & 32 & Composite & 4 & $\begin{array}{l}\text { Long standing abscess, sinus for 5 yrs. } \\
\text { Symptomatic h/o flare-up mid Tx }\end{array}$ & UR1 & RCT bleaching composite core \\
13 & F & 29 & - & 3 & $\begin{array}{l}\text { Trauma Lateral luxation Repositioned and splinted } \\
\text { Trauma sub-luxation Repositioned and splinted }\end{array}$ & LR2 & RCT composite core \\
14 & M & 17 & - & 3 & RCT Composite core \\
15 & M & 19 & - & 4 & Trauma avulsed surgically repositioned and splinted & LL3 & RCT bleaching composite build-up \\
\hline
\end{tabular}


joining $(\mathrm{NJ})$ method, based on 16S rRNA gene sequence comparisons, using the MEGA (version 4.1) program (http://www. megasoftware.net/).

\section{Phylotyping of $P$. acnes isolates}

Endodontic $P$. acnes isolates identified by partial $16 \mathrm{~S}$ rRNA gene sequencing were typed by partial $r e c A$ gene sequencing [18]. All selected $P$. acnes isolates were subcultured on FAA and grown for $24 \mathrm{~h}$.

The $P$. acnes recA gene was amplified using primer PAR-1 (positions -96 to -75 ; 5'-AGCTCGGTGGGGTTCTCTCATC$3^{\prime}$ ) and primer PAR-2 (positions +1105 to +1083 ; 5'GCTTCCTCATACCACTGGTCATC-3'), which generated a 1201-bp amplicon [18]. The reaction mix (final volume, $25 \mu \mathrm{l}$ ) comprised of $0.5 \mu \mathrm{l}$ of PAR-1 (concentration $10 \mathrm{pmol} / \mu \mathrm{l}$; Sigma), $0.5 \mu$ of PAR-2 (concentration $10 \mathrm{pmol} / \mu \mathrm{l}$; Sigma), $23 \mu \mathrm{l}$ of Reddymix (Thermo Scientific) and $1 \mu \mathrm{LNA}$ extract.

The thermal cycling conditions included initial denaturation at $95{ }^{\circ} \mathrm{C}$ for $3 \mathrm{~min}$, denaturation at $95{ }^{\circ} \mathrm{C}$ for $1 \mathrm{~min}$, annealing at $55^{\circ} \mathrm{C}$ for $30 \mathrm{~s}$, and extension at $72{ }^{\circ} \mathrm{C}$ for $90 \mathrm{~s}$, repeated for 35 cycles and a final extension at $72{ }^{\circ} \mathrm{C}$ for $10 \mathrm{~min}$. Amplified products were run on a $0.5 \%$ agarose gel and visualized under UV transillumination.

Sequencing was performed as described above. The $P$. acnes recA sequences were compared with GenBank sequences AY642055 (type IA), EU687255 (type IB), AY642061 (type II) and DQ672252 (type III). NJ trees were constructed using the Jukes-Cantor method with MEGA (version 4.1) software (www.megasoftware.net/).
Fig. 1 Prevalence of the 33 microbial taxa in 7 primary endodontic lesions without communications with the oral environment

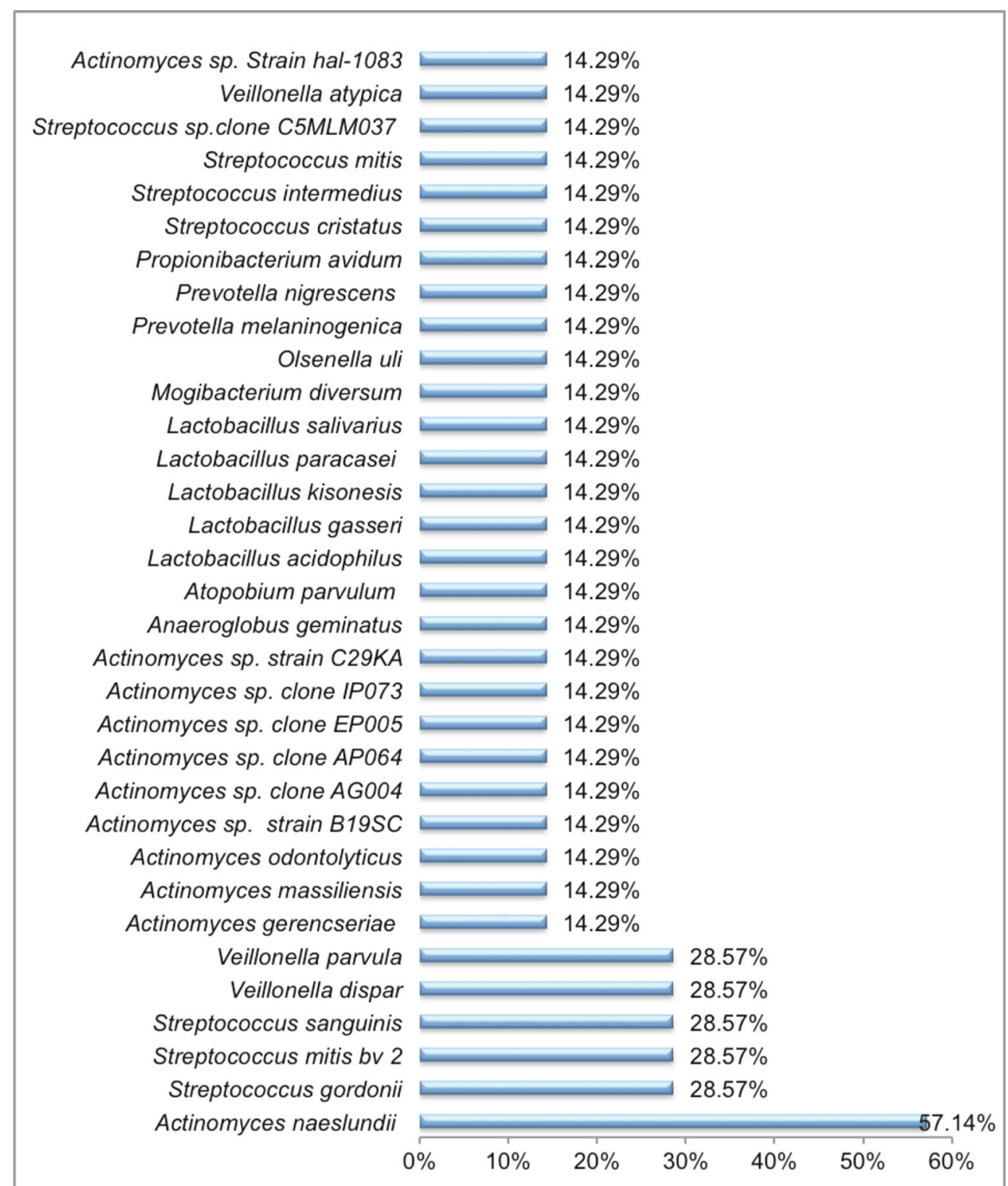




\section{Statistical analysis}

Data distributions were compared using $\chi 2$ tests; means were compared using the Mann-Whitney $U$ test in SPSSPC (Version 21. IBM, USA). Linear regression analysis was used to determine the effect of open or closed oral communication on the presence of $P$. acnes.

\section{Results}

No organisms were recovered from the samples taken from the disinfected tooth surfaces prior to making access into the root canal. The range of organisms cultured from the 15 primary endodontic infections is shown in Tables 1 and 2. From the 15 samples, a mean of $5.6 \pm 3.5$ taxa were detected. The number of species identified from lesions without communication $(5.86 \pm 3.7)$ was not different $(P>0.05)$ than the number from lesions with communication $(5.37 \pm 3.6)$.

\section{Cultivable taxa from primary endodontic infection without "open" communications with the oral cavity}

In the 7 primary endodontic infections without communication with the oral cavity, 33 cultivable bacterial taxa were detected amongst the 132 isolates recovered (Fig. 1). The microbiota of these samples were dominated by the Grampositive facultative anaerobes, which accounted for 23 of the 31 identified taxa. Furthermore, three Gram-positive obligate anaerobes including Atopobium parvulum, Mogibacterium diversum and Olsenella uli were also identified in these samples. Only a minimal number of Gram-negative bacteria
Fig. 2 Phylogenetic tree showing all 33 bacterial taxa belonging to 3 phyla from 132 isolates identified from the 7 primary endodontic cases without communications. The tree was constructed by the neighborjoining method based on $16 \mathrm{~S}$ rRNA gene sequence comparisons. The scale bar represents 0.05 substitutions per nucleotide position. The numbers at the node of the tree indicate bootstrap values for each node out of 500 bootstrap resampling

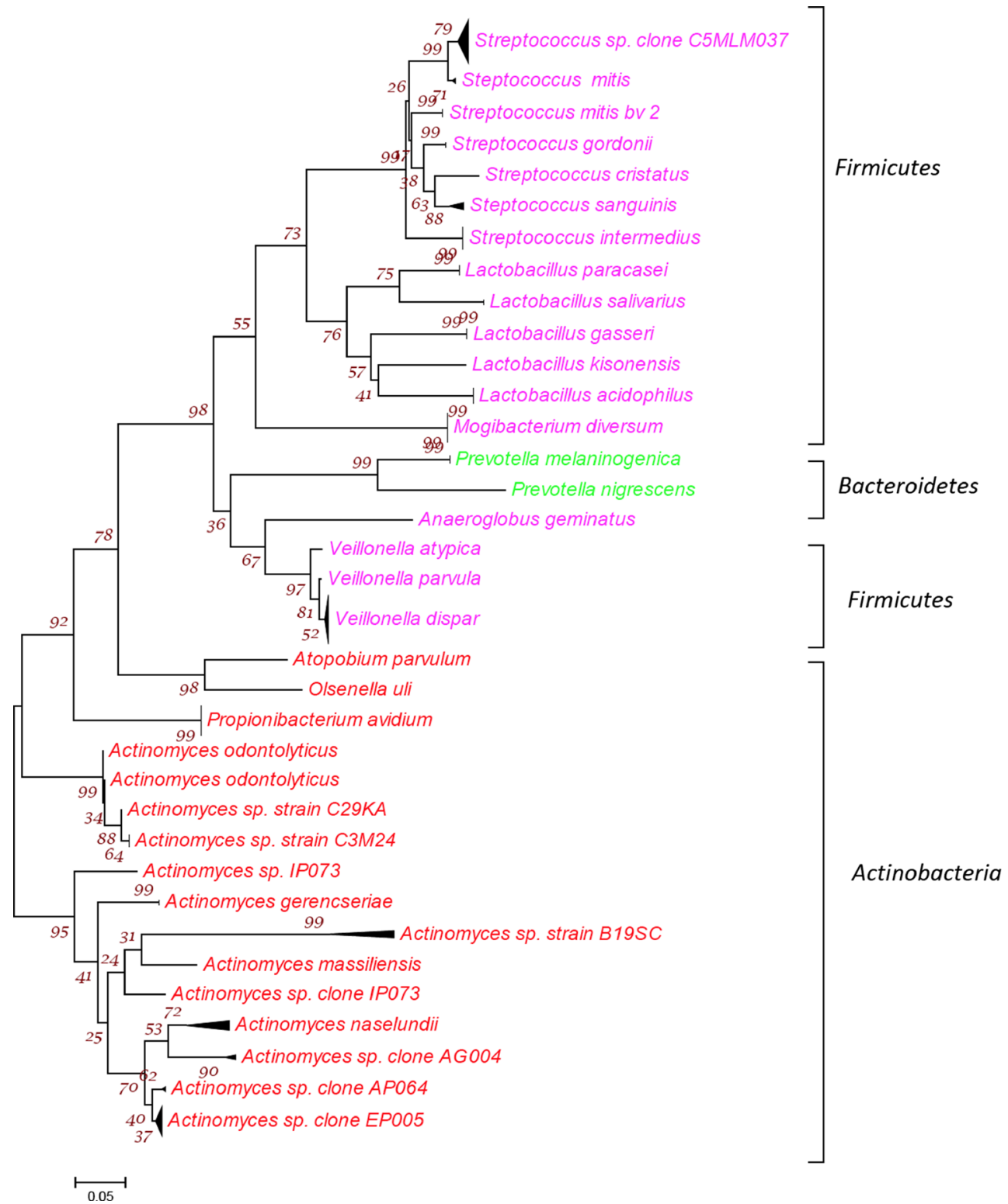


were identified in these primary endodontic cases, which comprised obligate anaerobes including two Prevotella species (Prevotella melaninogenica and P. nigrescens) and three species of Veillonella (Veillonella parvula, Veillonella dispar and Veillonella atypical) (Fig. 2). No Gram-negative facultative anaerobes were found in these samples. Actinomyces naeslundii was the most prevalent bacterial taxa present in $57 \%$ of the cases. This was followed by Streptococcus gordonii, Streptococcus mitis bv, Streptococcus sanguinis, $V$. dispar and V. parvula (28.57\% of cases), and all the rest of the taxa were present in $14.29 \%$.of the cases (Fig. 1).

\section{Cultivable taxa from primary endodontic infection with "open" communications with the oral cavity}

From the 8 primary endodontic infections presenting with oral communication, 26 bacterial taxa were detected amongst the 165 isolates recovered (Fig. 3). Microbiota of these infections was mainly dominated by the Gram-positive facultative anaerobes $(n=17)$ with $P$. acnes being the most prevalent bacterial taxa present in all cases (Fig. 3). Staphylococcus epidermidis was also recovered. Moreover, these lesions also harbored Gram-positive obligate anaerobes $(n=1)$ including Eubacterium $[X I][G-1]$ sulci and Gramnegative obligate anaerobes $(n=6)$ including one specie of Dialister (Dialister invisus,), two species of Fusobacterium (Fusobacterium nucleatum ss vincentii and Fusobacterium nucleatum ss Animalis) and three different species of Prevotella (Prevotella tannerae, Prevotella nigrescens and Prevotella $\mathrm{sp}$. oral strain B31FD) (Fig. 4).

$P$. acnes was recovered from all cases. Moreover, Streptococcus sp. clone C5MLM037 was recovered from $37.50 \%$ cases, followed by Actinomyces sp. clone AG004, Actinomyces sp. clone AP064, D. invisus, Eubacterium $[X I][G-1]$ sulci, F. nucleatum ss vincentii and Haemophilus parainfluenzae recovered from $25 \%$. All the rest of the taxa were present in $12.50 \%$ cases (Fig. 5).

\section{Comparison of the richness of bacterial taxa distributed in different phyla in primary endodontic lesions with and without communications with the oral environment}

The bacterial taxa identified from the seven primary cases with communication mainly belonged to three phyla including Firmicutes, Actinobacteria and Bacteroidetes. The Firmicutes
Fig. 3 Prevalence of the 26 microbial taxa in 8 primary endodontic lesions with communications with the oral environment

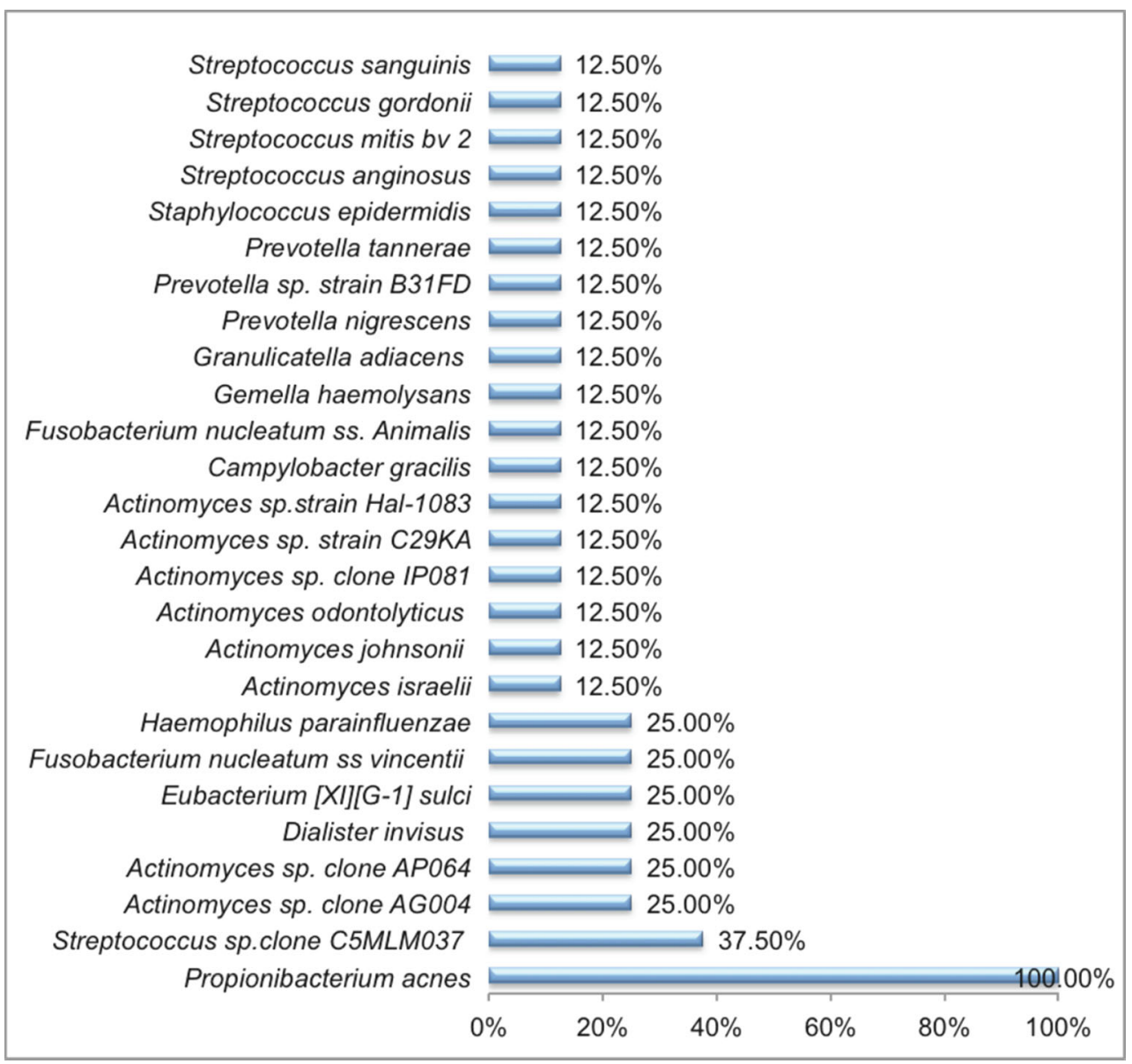


Fig. 4 Phylogenetic tree showing all 26 bacterial taxa belonging to 5 phyla from 136 isolates identified from the 8 primary endodontic cases with communications. The tree was constructed by the neighborjoining method based on $16 \mathrm{~S}$ rRNA gene sequence comparisons. The scale bar represents 0.05 substitutions per nucleotide position. The numbers at the node of the tree indicate bootstrap values for each node out of 500 bootstrap resampling.

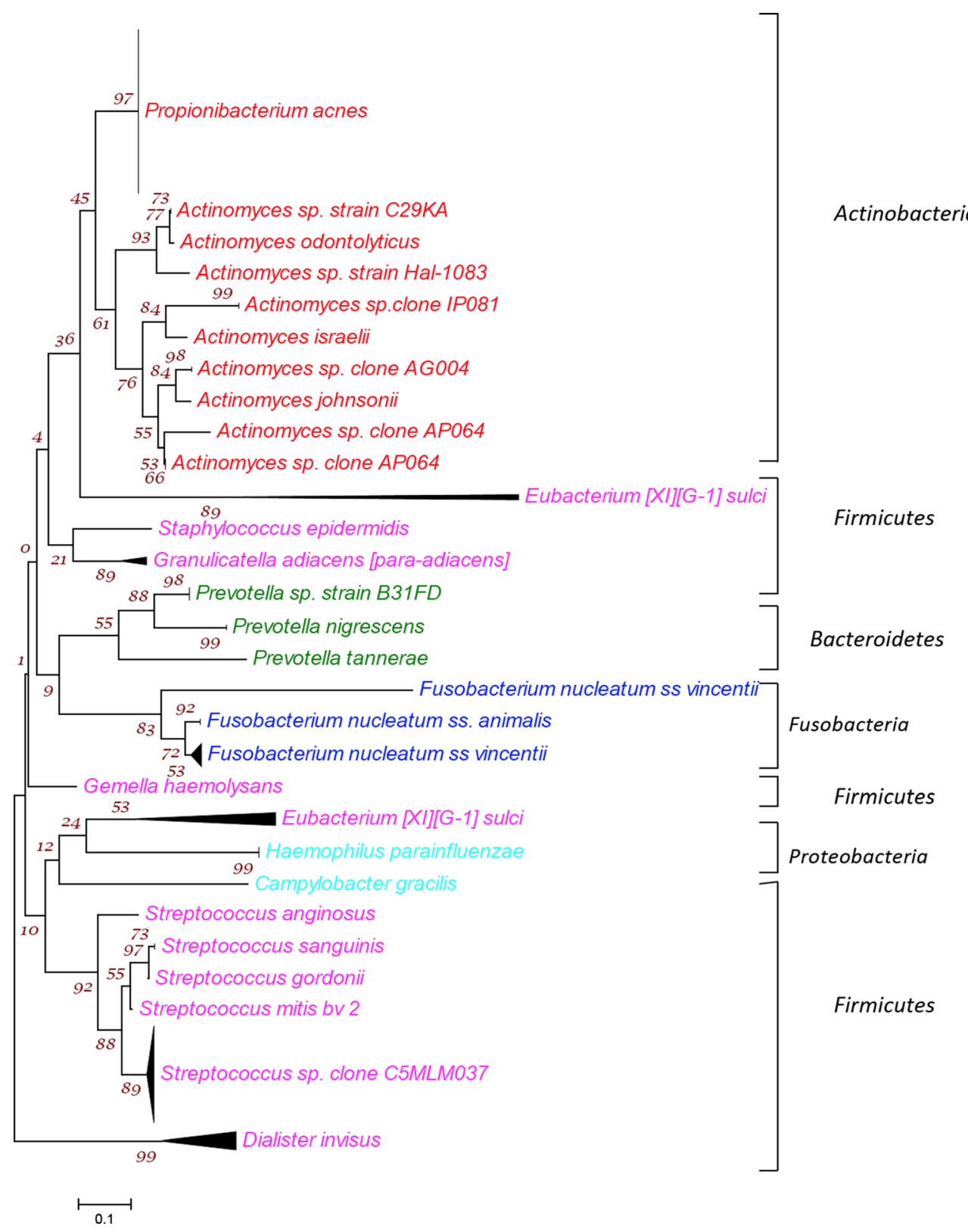

was the phylum with largest number of bacterial taxa that comprised of a variety of seven different species of Streptococci and five Lactobacilli species, in addition to Mogibacterium diversum, Anaeroglobus geminates along with three species of Veillonella. The next biggest phylum was Actinobacteria with 11 different Actinomyces species and one Propionibacteria including P. avidum, O. uli and A. parvulum. Bacteroidetes included two species of Prevotella (Fig. 2).

The bacterial taxa identified from the eight primary cases with communication belonged to five different phyla namely Firmicutes, Actinobacteria, Bacteroidetes, Proteobacteria and Fusobacteria (Fig. 4). The Firmicutes mainly included the Gram-positive anaerobes including five different species of Streptococci, S. epidermidis, Granulicatella adiacens, Gemella haemolysans and Eubacterium [XI][G-1] sulci, along with one Gram-negative anaerobe including $D$. invisus. The second richest phylum was Actinobacteria, which included P. acnes and nine different Actinomyces species. Phylum Bacteroidetes comprised of three species of Prevotella. Two species of Fusobacterium belonging to phylum Fusobacteria were also identified in the samples. Moreover, phylum Proteobacteria comprised of Campylobacter gracilis and H. parainfluenza (Fig. 4).

The richness of the bacterial taxa distributed in different phyla identified from primary endodontic lesions with and without communications is shown in Table 3. In Firmicutes phylum, the number of representatives is higher in the lesions 


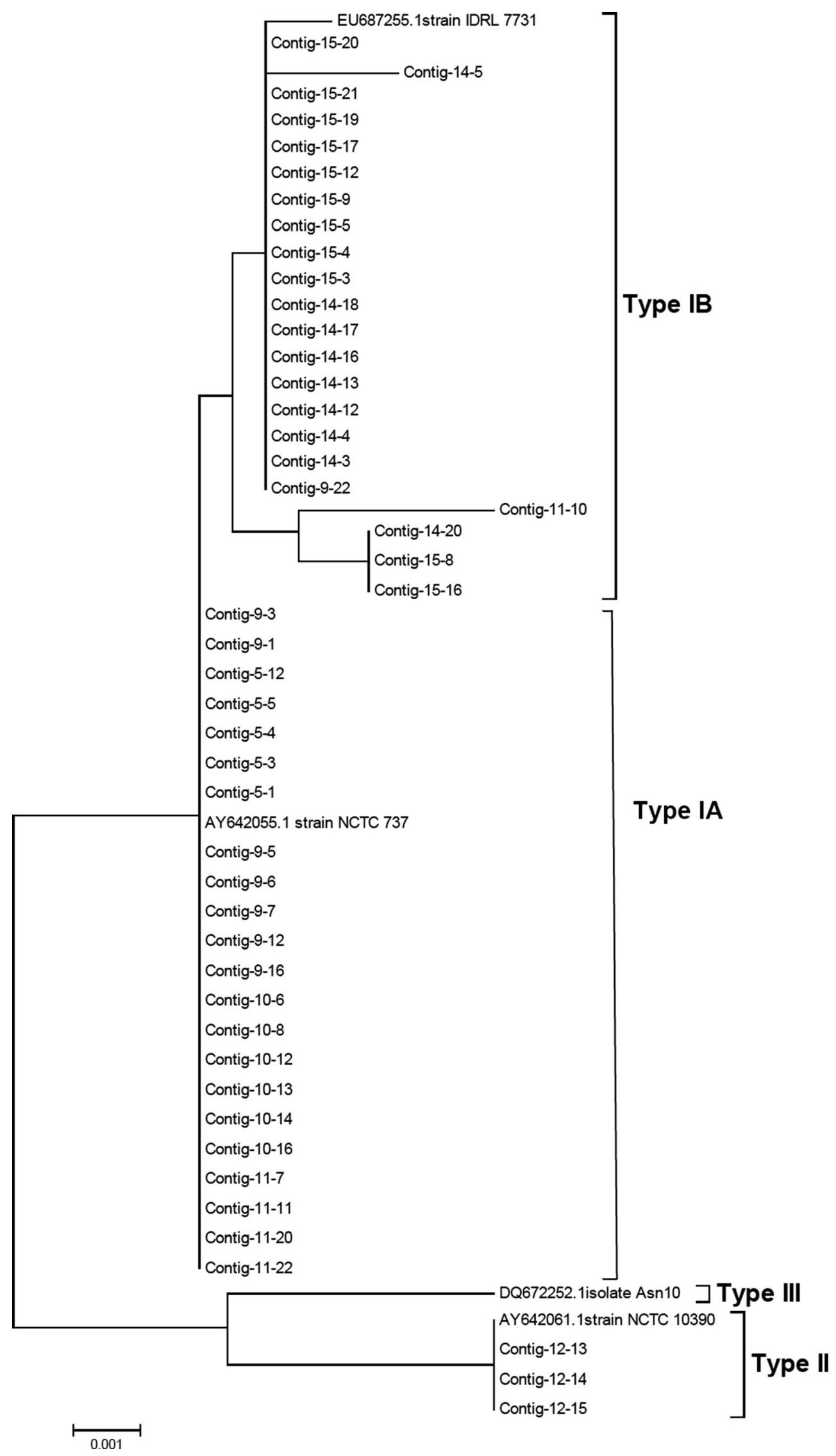


Fig. 5 Phylogenetic tree obtained by partial recA sequencing of 47 endodontic $P$. acnes isolates showing three distinct $P$. acnes phylotypes type IA, IB and II. Neighbor-joining $(N J)$ tree was constructed using the Jukes-Cantor method. The scale bar represents 0.05 substitutions per nucleotide position. The numbers at the node of the tree indicate bootstrap values for each node out of 500 bootstrap resampling

without communications as compared to the samples with communications. Proteobacteria and Fusobacteria were exclusively present in samples with communication, with two representatives in each phylum. The distributions were significantly different $(P<0.05)$. Linear regression analysis showed that open oral communication was a significant predictive value for the presence of $P$. acnes.

\section{Phylotyping of $P$. acnes isolates}

Partial recA sequences were obtained for 47 endodontic $P$. acnes isolates, and on the basis of sequence alignment, 2 distinct phylogenetic lineages, type I and type II, were observed. Type I was the dominant phylotype, comprising of 44 out of 47 of the endodontic $P$. acnes isolates. The type I isolates segregated into two distinct groups, into which the sequences from known type IA and type IB sequences clustered (Fig. 5).

\section{Discussion}

It is well established that microbial infection of the root canal system is the cause of apical periodontitis [29]. Previous studies by $16 \mathrm{~S}$ rRNA gene clone libraries demonstrated that 391 bacterial taxa belonging to 82 genera and 9 phyla have been identified in primary endodontic infections [30-33]. We used a culture-based approach to investigate the microbiota of primary endodontic infections with or without communications with the oral environment, along with further molecular identification by specific gene sequencing. With a culture-based approach, fewer taxa may be identified than are detectable with $16 \mathrm{~S}$ rRNA cloning or cloning combined with culture approaches. In a previous study of five primary endodontic lesions in four patients, a mean of 20.2 taxa were identified using a combined cloning and cultural approach [34], whereas a mean of 6.33 taxa was identified in this study. However, along with cataloging the taxa of these lesions, using culture-based approach we further investigated the phylotyping of the $P$. acnes isolates based on house-keeping gene (recA) sequencing (Table 4 and Table 5).

There are several avenues for the root canal to become infected. This includes dental caries with involvement of the pulp or as an infection following root canal treatment. It may also arise after a dental trauma. In some instances, there are no obvious cause(s) for root canal infection. In such cases, the crown and the root of the tooth may be intact [35]. All these situations influence the microbiota of endodontic lesions as either dominated by caries organisms or opportunistic bacteria. One of the consequences of trauma may also be vertical root fracture and formation of micro-cracks that may represent a further pathway for open contamination of the root canal space. In the present study, the signs of vertical root fracture were excluded under microscope magnification and in the absence of erratic electronic apex locator reading.

Although all sampled canals had radiographic signs of chronic apical periodontitis indicating a later stage of the disease process, the reported differences in the microbiota between the lesions with and without "open" communication with the oral cavity might be due to their differences in the root canal environment. The exposure of pulp space to the oral environment may allow the direct entry of bacteria, nutrients and oxygen into the root canal space, creating an environment that is radically different from that of root canals with no direct exposure to the oral environment such as those invaded by bacteria associated with caries where a low redox potential and lack of nutrients exist. It was therefore postulated that significant qualitative and quantitative differences in the microbiota might exist between exposed and unexposed canals.

Diverse groups of Gram-positive and Gram-negative bacteria have been identified from the cases included in this study. The taxa found exclusively in cases with communication include $P$. acnes, $S$. epidermidis, $F$. nucleatum ss vincentii, F. nucleatum ss animalis, $H$. influenza, C. gracilis and $D$. invisus. In cases without communication, a variety of Lactobacilli and Veillonella were exclusively found along with more species of Streptococcus as compared to the ones in cases with communication.
Table 3 Comparison of the bacterial phyla richness in primary endodontic lesions with and without communications with the oral environment

\begin{tabular}{lcc}
\hline Phyla & $\begin{array}{l}\text { Taxa detected in primary endodontic lesions } \\
\text { without communication }\end{array}$ & $\begin{array}{l}\text { Taxa detected in primary endodontic lesions } \\
\text { with communications }\end{array}$ \\
\hline Firmicutes & 17 & 10 \\
Actinobacteria & 14 & 10 \\
Bacteriodetes & 2 & 3 \\
Proteobacteria & - & 2 \\
Fusobacteria & - & 2 \\
\hline
\end{tabular}


Table 4 Distribution of all 26 bacterial taxa identified among 165 isolates recovered from 8 primary endodontic lesions with communications with oral environment

\begin{tabular}{|c|c|c|c|c|c|c|c|c|}
\hline \multirow{2}{*}{$\frac{\text { Organism }}{\text { Gram-positive organisms }}$} & \multicolumn{8}{|c|}{ Presence in primary endodontic lesions with communication } \\
\hline & 8 & 9 & 10 & 11 & 12 & 13 & 14 & 15 \\
\hline \multicolumn{9}{|l|}{ Obligate anaerobes } \\
\hline Eubacterium $[X I][G-1]$ sulci & - & - & - & - & + & + & - & - \\
\hline \multicolumn{9}{|l|}{ Facultative anaerobes } \\
\hline Actinomyces israelii & + & - & - & - & - & - & - & - \\
\hline Actinomyces johnsonii & + & - & - & - & - & - & - & - \\
\hline Actinomyces odontolyticus & + & - & - & - & - & - & - & - \\
\hline Actinomyces sp. clone AG004 & - & + & - & + & - & - & - & - \\
\hline Actinomyces sp. clone AP064 & + & - & - & - & - & + & - & - \\
\hline Actinomyces sp. clone IP081 & + & - & - & - & - & - & - & - \\
\hline Actinomyces sp. strain C29KA & - & - & - & - & - & - & + & - \\
\hline Actinomyces sp. strain Hal-1083 & + & - & - & - & - & - & - & - \\
\hline Gemella haemolysans & - & - & - & - & - & - & + & - \\
\hline Granulicatella adiacens & - & - & - & - & - & - & - & + \\
\hline Propionibacterium acnes & + & + & + & + & + & + & + & + \\
\hline Staphylococcus epidermidis & - & - & - & - & + & - & - & - \\
\hline Streptococcus anginosus & - & - & - & - & - & + & - & - \\
\hline Streptococcus gordonii & + & - & - & - & - & - & - & - \\
\hline Streptococcus mitis by 2 & + & - & - & - & - & - & - & - \\
\hline Streptococcus sanguinis & - & - & - & - & - & - & + & - \\
\hline Streptococcus sp. clone C5MLM037 & - & + & + & + & - & - & - & - \\
\hline \multicolumn{9}{|l|}{ Gram-negative organisms } \\
\hline \multicolumn{9}{|l|}{ Obligate anaerobes } \\
\hline Dialister invisus & - & - & - & - & + & + & - & - \\
\hline Fusobacterium nucleatum ss vincentii & - & - & - & - & + & + & - & - \\
\hline \multicolumn{9}{|l|}{ Fusobacterium nucleatum ss } \\
\hline Animalis & + & - & - & - & - & - & - & - \\
\hline Prevotella nigrescens & + & - & - & - & - & - & - & - \\
\hline Prevotella sp. strain B31FD & - & - & - & - & + & - & - & - \\
\hline Prevotella tannerae & + & - & - & - & - & - & - & - \\
\hline \multicolumn{9}{|l|}{ Facultative anaerobes } \\
\hline Campylobacter gracilis & + & - & - & - & - & - & - & - \\
\hline Haemophilus parainfluenzae & - & - & - & - & - & - & + & + \\
\hline
\end{tabular}

Among the Phyla Firmicutes, D. invisus was only found in cases with communication. The high prevalence of $D$. invisus was also reported in other studies [36, 37]; their presence is dictated by the ideal environment of an abscess.
Fusobacterium is also associated with the lesions with abscesses. F. nucleatum has been reported to be associated with the more severe form of inter-appointment flare-ups [38]. In this study, there were significant differences between the two
Table 5 Comparison of the bacterial phyla richness in primary endodontic lesions with and without communications with the oral environment

\begin{tabular}{lcc}
\hline Phyla & $\begin{array}{c}\text { Taxa detected in primary endodontic } \\
\text { lesions without communication }\end{array}$ & $\begin{array}{l}\text { Taxa detected in primary endodontic } \\
\text { lesions with communications }\end{array}$ \\
\hline Firmicutes & 17 & 10 \\
Actinobacteria & 14 & 10 \\
Bacteriodetes & 2 & 3 \\
Proteobacteria & - & 2 \\
Fusobacteria & - & 2 \\
\hline
\end{tabular}


cohorts in the prevalence of $F$. nucleatum and $H$. parainfluenza being isolated from approximately $25 \%$ of the communication lesions.

P. acnes was the most prevalent bacterial taxa found in all the primary endodontic cases with communication. However, $P$. acnes was not recovered from the cases without communication. This emphasizes the ability of $P$. acnes to gain access through the possible communications into the root canal space and then establish itself as a predominant microbiota of such cases. A previous study showed the presence of $P$. acnes and $S$. epidermidis in the samples from refractory endodontic infections [25]. Other studies that recovered P. acnes and $S$. epidermidis considered it as contaminants since they are skin commensals [3, 18, 24, 39, 40]. In a previous study by Niazi et al. (2010), a decontamination protocol was used to remove contaminating organisms from the tooth surface prior to entering the diseased tooth [25]. Similar decontamination protocol was used in this study, thus confirming that $P$. acnes recovered from these primary cases with communication were opportunistic endodontic pathogens rather than contaminants, which is partially confirmed by the lack of $P$. acnes isolated in teeth with no communication with the oral cavity.

P. acnes has several virulence factors, in particular protease and polysaccharide formation capabilities are linked to chronic or persistent low-grade implant-associated infections. In these subclinical scenarios, in the absence of positive cultures, this pathogen is probably under-recognized and underestimated [41].

The genotypic analysis of the $P$. acnes isolates using recA gene sequencing showed that type IA and IB were the prevalent phylotypes found in primary cases with communication. These phylotypes probably were derived from patient's own skin or oral cavity during the exposure and communication of these teeth through the sinus tract, periodontal involvement or root fracture. These might also be the result of a nosocomial infection occurred as a result of manipulation of these teeth during treatment $[25,42]$. Type II phylotype of $P$. acnes was only indentified in one case collected from the teeth with a long-standing infections ( $>5$ years) and a history of intermittent flare-ups. Although, the exact source of type II is still not identified, there could be a relationship between the occurrence of these phylotypes and the presence of long-standing infections with "open" communication with the oral cavity. This speculation is also partially supported by the more frequent isolation of philotypes II and III in failed root canal treatment cases (25) which are likely to be associated with longer standing infections.

P. acnes is the most prevalent species in primary endodontic infections with a history or clinical evidence of communication with the oral environment, whereas it is absent in lesions without communications. Using recA sequencing, this study showed that $P$. acnes type IA and $1 \mathrm{~B}$ are associated with primary endodontic infections with communications. P. acnes in primary endodontic infections with communications with the oral cavity are likely to act as opportunistic pathogens.

The clinical management of endodontic cases where an open lesion is present may require specific strategies to maximize the chances to eliminate the opportunistic pathogens, such as $P$. acnes. In particular, adopting two stages root canal treatment approach with usage of intermediate dressing may be required even in primary endodontic treatment, where single-stage endodontics was usually considered.

Acknowledgments The project received financial support from The Department of Health via the National Institute for Health Research (NIHR) Comprehensive Biomedical Research Centre award to Guy's \& St Thomas' NHS Foundation Trust in partnership with King's College London and King's College Hospital NHS Foundation Trust.

Compliance with ethical standards The project was approved by the local ethics committee (South London REC: 05/Q0705/051).

Conflict of interest The authors declare that they have no competing interests.

Informed consent The patient had verbal and written information about the purpose of study and they gave their written informed consent prior to their inclusion.

Open Access This article is distributed under the terms of the Creative Commons Attribution 4.0 International License (http:// creativecommons.org/licenses/by/4.0/), which permits unrestricted use, distribution, and reproduction in any medium, provided you give appropriate credit to the original author(s) and the source, provide a link to the Creative Commons license, and indicate if changes were made.

\section{References}

1. Tancrede C (1992) Role of human microflora in health and disease. Eur J Clin Microbiol Infect Dis 11(11):1012-1015

2. McDowell A, Gao A, Barnard E, Fink C, Murray PI, Dowson CG, Nagy I, Lambert PA, Patrick S (2011) A novel multilocus sequence typing scheme for the opportunistic pathogen Propionibacterium acnes and characterization of type I cell surface-associated antigens. Microbiology 157(Pt 7):1990-2003. doi:10.1099/mic.0.049676-0

3. Brook I, Frazier EH (1991) Infections caused by Propionibacterium species. Rev Infect Dis 13(5):819-822

4. Doyle A, Beigi B, Early A, Blake A, Eustace P, Hone R (1995) Adherence of bacteria to intraocular lenses: a prospective study. $\mathrm{Br}$ J Ophthalmol 79(4):347-349

5. Eady EA, Ingham E (1994) Propionibacterim acnes-friend or foe? Rev Med Microbiol 5:163-173

6. Leyden JJ (2001) The evolving role of Propionibacterium acnes in acne. Semin Cutan Med Surg 20(3):139-143

7. Eishi Y, Suga M, Ishige I, Kobayashi D, Yamada T, Takemura T, Takizawa T, Koike M, Kudoh S, Costabel U, Guzman J, Rizzato G, Gambacorta M, du Bois R, Nicholson AG, Sharma OP, Ando M (2002) Quantitative analysis of mycobacterial and propionibacterial DNA in lymph nodes of Japanese and European patients with sarcoidosis. J Clin Microbiol 40(1):198-204

8. Perry AL, Lambert PA (2006) Propionibacterium acnes. Lett Appl Microbiol 42(3):185-188 
9. Schaeverbeke T, Lequen L, de Barbeyrac B, Labbe L, Bebear CM, Morrier Y, Bannwarth B, Bebear C, Dehais J (1998) Propionibacterium acnes isolated from synovial tissue and fluid in a patient with oligoarthritis associated with acne and pustulosis. Arthritis Rheum 41(10):1889-1893

10. Cohen RJ, B.A. S, J.E. MN, Shannon T, K.L. G (2005) Propionibacterium acnes associated with inflammation in radical prostatectomy specimens: a possible link to cancer evolution? J Urol 173:1969-1974

11. Mathisen GE, D. MR, L. GW, M. CD (1984) Brain abscess and cerebritis. Rev Infect Dis 6(Suppl.1):101-106

12. Abolnik IZ, Eaton JV, Sexton DJ (1995) Propionibacterium acnes vertebral osteomyelitis following lumbar puncture: case report and review. Clin Infect Dis 21(3):694-695

13. Harris AE, Hennicke C, Byers K, Welch WC (2005) Postoperative discitis due to Propionibacterium acnes: a case report and review of the literature. Surg Neurol 63(6):538-541 discussion 541

14. Halkic N, Blanc C, Corthesy ME, Corpataux JM (2001) Lumbar spondylodiscitis after epidural anaesthesia at a distant site. Anaesthesia 56(6):602-603

15. Friberg O, Svedjeholm R, Kallman J, Soderquist B (2007) Incidence, microbiological findings, and clinical presentation of sternal wound infections after cardiac surgery with and without local gentamicin prophylaxis. Eur J Clin Microbiol Infect Dis 26(2):91-97

16. Benz MS, Scott IU, Flynn HW Jr, Unonius N, Miller D (2004) Endophthalmitis isolates and antibiotic sensitivities: a 6-year review of culture-proven cases. Am J Ophthalmol 137(1):38-42

17. Gunthard H, Hany A, Turina M, Wust J (1994) Propionibacterium acnes as a cause of aggressive aortic valve endocarditis and importance of tissue grinding: case report and review. J Clin Microbiol 32(12):3043-3045

18. McDowell A, Valanne S, Ramage G, Tunney MM, Glenn JV, McLorinan GC, Bhatia A, Maisonneuve JF, Lodes M, Persing DH, Patrick S (2005) Propionibacterium acnes types I and II represent phylogenetically distinct groups. J Clin Microbiol 43(1):326334

19. McDowell A, Perry AL, Lambert PA, Patrick S (2008) A new phylogenetic group of Propionibacterium acnes. J Med Microbiol 57(Pt 2):218-224

20. Valanne S, McDowell A, Ramage G, Tunney MM, Einarsson GG, O'Hagan S, Wisdom GB, Fairley D, Bhatia A, Maisonneuve JF, Lodes M, Persing DH, Patrick S (2005) CAMP factor homologues in Propionibacterium acnes: a new protein family differentially expressed by types I and II. Microbiology 151(Pt 5):1369-1379

21. Lodes MJ, Secrist H, Benson DR, Jen S, Shanebeck KD, Guderian J, Maisonneuve JF, Bhatia A, Persing D, Patrick S, Skeiky YA (2006) Variable expression of immunoreactive surface proteins of Propionibacterium acnes. Microbiology 152(12):3667-3681

22. Nagy I, Pivarcsi A, Kis K, Koreck A, Bodai L, McDowell A, Seltmann H, Patrick S, Zouboulis CC, Kemeny L (2006) Propionibacterium acnes and lipopolysaccharide induce the expression of antimicrobial peptides and proinflammatory cytokines/ chemokines in human sebocytes. Microbes and Infection/Institut Pasteur 8(8):2195-2205. doi:10.1016/j.micinf.2006.04.001

23. McDowell A, Barnard E, Nagy I, Gao A, Tomida S, Li H, Eady A, Cove J, Nord CE, Patrick S (2012) An expanded multilocus sequence typing scheme for Propionibacterium acnes: investigation of 'pathogenic', 'commensal' and antibiotic resistant strains. PLoS One 7(7):e41480. doi:10.1371/journal.pone.0041480

24. Sampedro MF, Piper KE, McDowell A, Patrick S, Mandrekar JN, Rouse MS, Steckelberg JM, Patel R (2009) Species of Propionibacterium and Propionibacterium acnes phylotypes associated with orthopedic implants. Diagn Microbiol Infect Dis 64(2):138-145 doi:S0732-8893(09)00029-7

25. Niazi SA, Clarke D, Do T, Gilbert SC, Mannocci F, Beighton D (2010) Propionibacterium acnes and Staphylococcus epidermidis isolated from refractory endodontic lesions are opportunistic pathogens. J Clin Microbiol 48(11):3859-3869. doi:10.1128/jcm. 01326-10

26. Narayanan LL, Vaishnavi C (2010) Endodontic microbiology. J Conserv Dent 13(4):233-239. doi:10.4103/0972-0707.73386

27. Patel S, Wilson R, Dawood A, Foschi F, Mannocci F (2012) The detection of periapical pathosis using digital periapical radiography and cone beam computed tomography - part 2: a 1-year post-treatment follow-up. Int Endod J 45(8):711-723. doi:10.1111/j.13652591.2012.02076.x

28. Lane DJ (ed) (1991) 16S/23S rRNA sequencing. In nucleic acid techniques in bacterial systematics. Wiley, Chichester

29. Kakehashi S, Stanley HR, Fitzgerald RJ (1965) The effects of surgical exposures of dental pulps in germ-free and conventional laboratory rats. Oral Surg Oral Med Oral Pathol 20:340-349

30. Siqueira JF Jr, Rocas IN (2009) Diversity of endodontic microbiota revisited. J Dent Res 88(11):969-981

31. Lee YJ, Kim MK, Hwang HK, Kook JK (2005) Isolation and identification of bacteria from the root canal of the teeth diagnosed as the acute pulpitis and acute periapical abscess. J Korean Acad Conserv Dent 30(5):13

32. Kim SY, Choi HY, Park SH, Choi B (2003) Distribution of oral pathogens in infection of endodontic origin. J Korean Acad Conserv Dent 28(4):303-313

33. Kum KY, Foud AF (2003) PCR-based identification of Eubacteirum species in endodontic infection. J Korean Acad Conserv Dent 28(3):241-248

34. Munson MA, Pitt-Ford T, Chong B, Weightman A, Wade WG (2002) Molecular and cultural analysis of the microflora associated with endodontic infections. J Dent Res 81(11):761-766

35. van Steenbergen TJ, van Winkelhoff AJ, Mayrand D, Grenier D, de Graaff J (1984) Bacteroides endodontalis sp. nov., an asaccharolytic black-pigmented Bacteroides species from infected dental root canals. Int J Sys Bact 34:118-120

36. Fouad AF, Barry J, Caimano M, Clawson M, Zhu Q, Carver R, Hazlett K, Radolf JD (2002) PCR-based identification of bacteria associated with endodontic infections. J Clin Microbiol 40(9): 3223-3231

37. Siqueira JF Jr, Rocas IN (2002) Dialister pneumosintes can be a suspected endodontic pathogen. Oral Surg Oral Med Oral Pathol Oral Radiol Endod 94(4):494-498

38. LE C d PV (2002) Fusobacterium nucleatum in endodontic flareups. Oral Surg Oral Med Oral Pathol Oral Radiol Endod 93(2):179 183

39. Tunney MM, Patrick S, Gorman SP, Nixon JR, Anderson N, Davis RI, Hanna D, Ramage G (1998) Improved detection of infection in hip replacements. A currently underestimated problem. J Bone Joint Surg Br Volume 80(4):568-572

40. Lazar JM, Schulman DS (1992) Propionibacterium acnes prosthetic valve endocarditis: a case of severe aortic insufficiency. Clin Cardiol 15(4):299-300

41. Achermann Y, Goldstein EJ, Coenye T, Shirtliff ME (2014) Propionibacterium acnes: from commensal to opportunistic biofilm-associated implant pathogen. Clin Microbiol Rev 27(3): 419-440. doi:10.1128/CMR.00092-13

42. Patel S, Wilson R, Dawood A, Mannocci F (2012) The detection of periapical pathosis using periapical radiography and cone beam computed tomography - part 1: pre-operative status. Int Endod J 45(8):702-710. doi:10.1111/j.1365-2591.2011.01989.x 